\section{Medical Principles and Practice}

Med Princ Pract 2012;21:98

DOI: $10.1159 / 000334692$

\title{
The State of Kuwait Sponsors Publication of the English Translation of the Classic Latin Text De humani corporis fabrica by Andreas Vesalius
}

Karger Publishers will publish an English translation of De humani corporis fabrica (On the Fabric of the Human Body), with original commentary on and a comparison between the 1543 and 1555 editions of the work. Thanks to sponsorship from the State of Kuwait, this masterpiece of the Renaissance is being made accessible and modernized for science worldwide.

Andreas Vesalius (1514-1564) was one of the founding fathers of modern anatomy. At the age of 28 years, he published an atlas of anatomy in Latin containing illustrations that are still known to every student of medicine and most students of art. A second edition, revised by Vesalius himself, was published 12 years later.

In Vesalius' time, Basel was at the heart of the world of printing. Johannes Oporinus had printed and published both editions, which were about 700 pages long even in the book's large format. Vesalius himself guided the printing process. During that time, he also held a colloquium on anatomy that resulted in the preparation of a skeleton that can still be seen today at Basel's Museum of Anatomy.

Daniel H. Garrison and Malcolm H. Hast, two professors from Northwestern University in Chicago, Ill., USA, worked for more than 20 years on the English translation of the Fabrica, together with a small team, comparing the versions word for word, adding comments to clarify any deviations between the versions, and applying the common anatomical nomenclature according to the Nomina Anatomica still used today. In addition, they extracted and highlighted salient elements of the texts and illustrations for all readers, whether specialists or interested laypeople.

This English-language edition will be produced in A3 format $(297 \times 420 \mathrm{~mm})$, comprising approximately 1,400 pages and weighing about 14 kilograms. A detailed conceptual framework including a color scheme will help guide readers in a clear and simple way through the immense work and the translators' commentaries.

Such an ambitious project would not be possible without funding from both public and private sectors. Financial support from the State of Kuwait will provide Karger Publishers, an internationally active biomedical publishing house based in Basel, with the means of producing this meaningful work.

At a ceremony held in Basel on September 5, 2011, His Excellency the Minister of Health, Dr. Hilal Al-Sayer - accompanied by Professor Farida Al-Awadi, Senior Consultant, Minister's Office, and former Editor-in-Chief of Medical Principles and Practice - presented a generous donation from the State of Kuwait to the Basel publisher Thomas Karger for the publication of this English translation. Dr. Al-Sayer said of this generous donation:

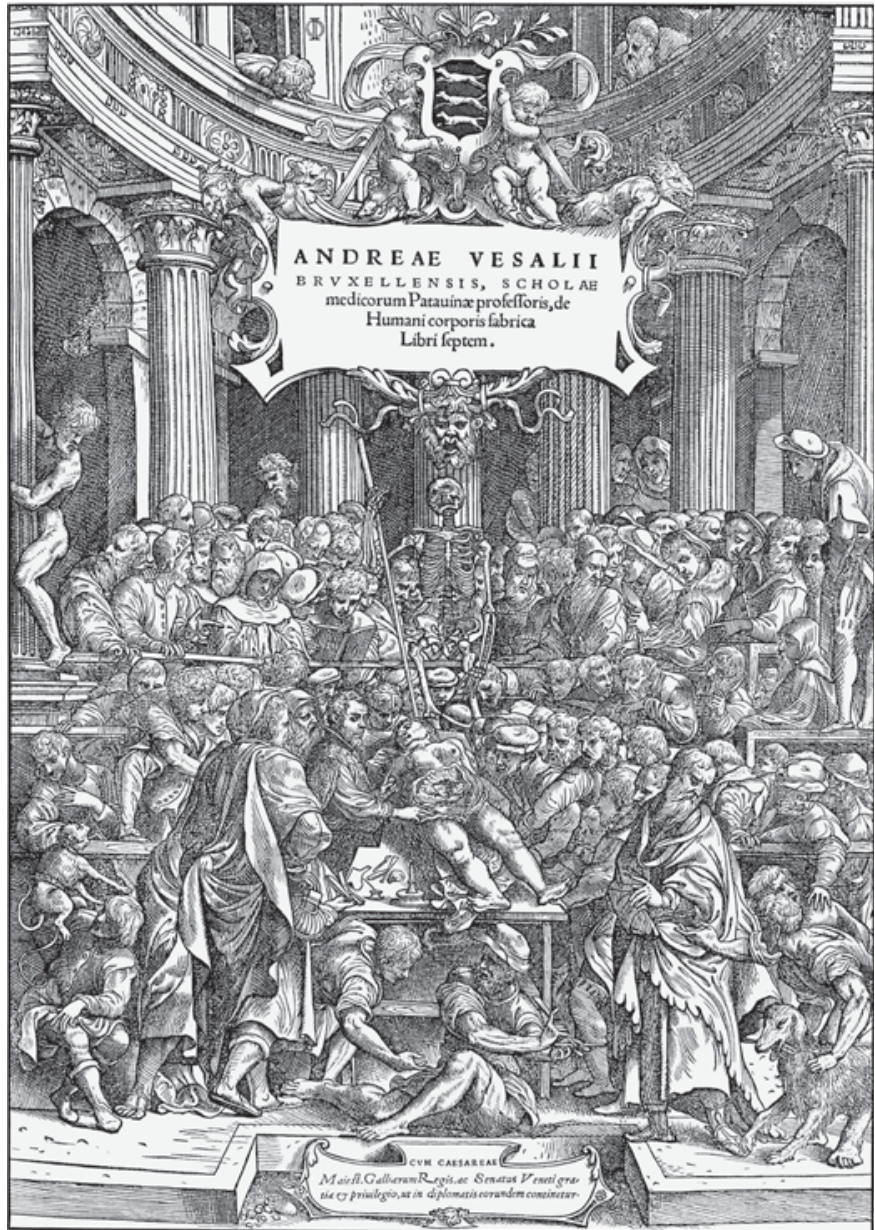

Image reproduction of the frontispiece of the Fabrica.

'Our main mission is to highlight Kuwait's contribution to the world in different areas of culture and academia. Our motive for sponsorship is purely altruistic in order to expedite the publication of this noble work.'

Karger Publishers will release the English translation of $D e$ humani corporis fabrica in no more than 2 years, in time to commemorate the 500th birthday of Andreas Vesalius in 2014. 